EPJ Web of Conferences 35, 02003 (2012)

DOI: $10.1051 /$ epjconf/20123502003

(C) Owned by the authors, published by EDP Sciences, 2012

\title{
Metallisation of single crystal diamond radiation detectors
}

\author{
Lucas Ong ${ }^{\mathrm{a}}$, Kumaravelu Ganesan, Andrew Alves, and Elisabetta Barberio
}

The University of Melbourne

\begin{abstract}
Properties such as a large band gap, high thermal conductivity and resistance to radiation damage make diamond an extremely attractive candidate for detectors in next generation particle physics experiments. This paper presents our technique for metallisation of a single crystal diamond grown by chemical vapour deposition (CVD) for use as a radiation detector, suitable for operation in places such as the Large Hadron Collider. The front and back side of the diamond are metalised with aluminium and gold on top of titanium respectively, after which the diamond is mounted and read out via a charge sensitive preamplifier. The device is found to collect charge at an efficiency of $97 \%$.
\end{abstract}

\section{Introduction}

Detectors and radiation monitors at high energy particle physics experiments are required to operate under harsh environments. These devices need to be exceedingly tolerant of radiation damage as it is often impossible to replace the innermost detectors in a timely and cost effective manner. Currently silicon remains the preferred material for high precision charged particle tracking sensors at the Large Hadron Collider (LHC). However, after the planned upgrade of the LHC, experiments will demand components capable of withstanding far higher levels of radiation.

In particular radiation levels will rise significantly when the Super LHC (SLHC) delivers an increased instantaneous luminosity, greater than before by an at least an order of magnitude [1]. As a consequence new types of tracking detectors capable of coping with hadron fluences of up to $10^{16} \mathrm{~cm}^{-2}$ and expected dose of $4200 \mathrm{kGy}$ must be developed. Alongside radiation hard silicon, diamond provides a suitable candidate for the next generation of tracking detectors [2].

Unlike traditional Si detectors, diamond posses a large band gap, high thermal conductivity and is exceedingly resistant to radiation damage [3] as shown in Table 1. This can result in efficient, radiation hard devices capable of room temperature operation. For experiments such as ATLAS (A Toroidal LHC ApparatuS) this represents a significant reduction in the complexity of cooling infrastructure and power consumption.

In this work we present the metallisation and fabrication of a single crystal CVD diamond detector. In addition, we measure the charge collection efficiency of the device using $3 \mathrm{MeV} \mathrm{H}^{+}$ions.

\footnotetext{
a e-mail: lucaso@unimelb.edu.au
}

Table 1. Comparison of the relevant properties between diamond and silicon at $293 \mathrm{~K}$ [4-6].

\begin{tabular}{lll}
\hline Property & Diamond & Silicon \\
\hline Density $\left(\mathrm{g} \mathrm{cm}^{-3}\right)$ & 3.5 & 2.33 \\
Bandgap $(\mathrm{eV})$ & 5.47 & 1.12 \\
Restivity $(\Omega)$ & $>10^{12}$ & $2.23 \times 10^{5}$ \\
Breakdown voltage $\left.\left(V \mathrm{~cm}^{-1}\right)\right)$ & $10^{7}$ & $3 \times 10^{5}(\mathrm{pn})$ \\
Electron mobility, $\mu_{\mathrm{e}}\left(\mathrm{em}^{2} V \mathrm{~s}^{-1}\right)$ & 2400 & 13350 \\
Hole mobility, $\mu_{\mathrm{h}}\left(\mathrm{em}^{2} V \mathrm{~s}^{-1}\right)$ & 2100 & 480 \\
e-h pair creation energy $(\mathrm{eV})$ & 13 & 3.6 \\
Thermal conductivity $\left(\mathrm{W} \mathrm{m} \mathrm{K}^{-1} \mathrm{~K}^{-1}\right)$ & $\sim 2000$ & 150 \\
\hline
\end{tabular}

\section{Metallisation}

Metallisation is preformed on an ultra high purity (UHP), electronics grade, CVD single crystal diamond provided by Element 6 . The sample measures $3.5 \mathrm{~mm} \mathrm{x} 3.5 \mathrm{~mm} x$ $0.5 \mathrm{~mm}$ and Figure 1 provides a schematic depiction of the geometry of the sample and the metallisation.

\subsection{Metallisation of the back side}

First, a thin $20 \mathrm{~nm}$ layer of titanium is created on the rear side of the sample using electron beam physical vapour deposition. A subsequent layer of gold, $100 \mathrm{~nm}$ thick, is then deposited on top of the titanium. In the way we create a robust contact with good electrical connection to the diamond. Annealing is performed at $800^{\circ} \mathrm{C}$ for 30 minutes, resulting in an ohmic junction at the interface between readout and diamond.

\subsection{Metallisation of the top side}

The top contact consists of an aluminium grid structure with each bar measuring $200 \mathrm{~nm}$ thick and $10 \mu \mathrm{m}$ wide 


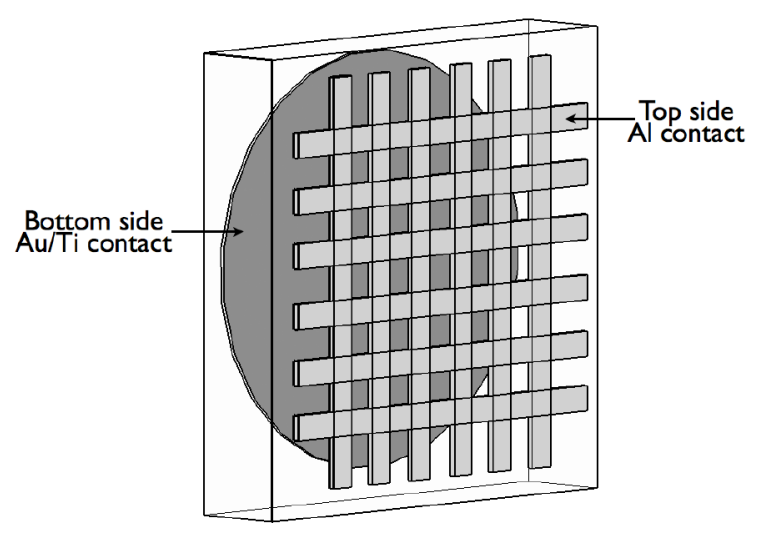

Fig. 1. Schematic diagram of the metalization.

and each internal square $300 \mu \mathrm{m} \times 300 \mu \mathrm{m}$. Again, the aluminium is deposited by electron beam physical vapour deposition and the structure created through photolithography. The top contact is not annealed.

\subsection{Mounting}

The device is mounted into a standard chip carrier. Contact with the back connection is made using sliver paste and the top connection is wire bonded to the pins of the chip carrier, shown in Figure 2. The device is read out using an ORTEC $142 \mathrm{~A}$ charge sensitive preamplifier.

\section{Charge collection efficiency}

Using a National Electrostatics Corporation 5U Pelletron Accelerator, located at the University of Melbourne, we measure how efficiently the charge induced by $3 \mathrm{MeV} \mathrm{H}^{+}$ ions is collected. The device is held at a bias of $100 \mathrm{~V}$ and wired according to Figure 3. The total charge induced within the diamond is $Q=e E_{H^{+}} / \epsilon$ where $\epsilon=13 \mathrm{eV}$ is the energy required to create an electron hole pair in diamond. The response of the preamplifier is shaped by an $O R T E C$ 570 pulse shaping amplifier and connected to a multichannel analyser. The setup is calibrated using a Hamamatsu silicon photodiode. At a bias voltage of $100 \mathrm{~V}$, the device collects charge with a mean efficiency of $97 \%$ over the non metalised surface. [7] contains further details on the analysis of the device.

\section{Conclusion}

Using electron beam physical vapour deposition and photolithography we have created a device from single crystal UHP CVD diamond capable of operation at $97 \%$ charge collection efficiency, suitable for radiation detection.

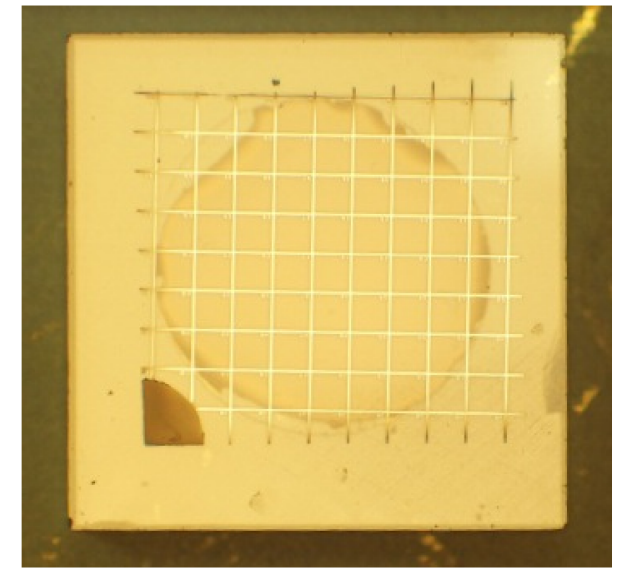

Fig. 2. Metalized sample after mounting into a chip carrier.

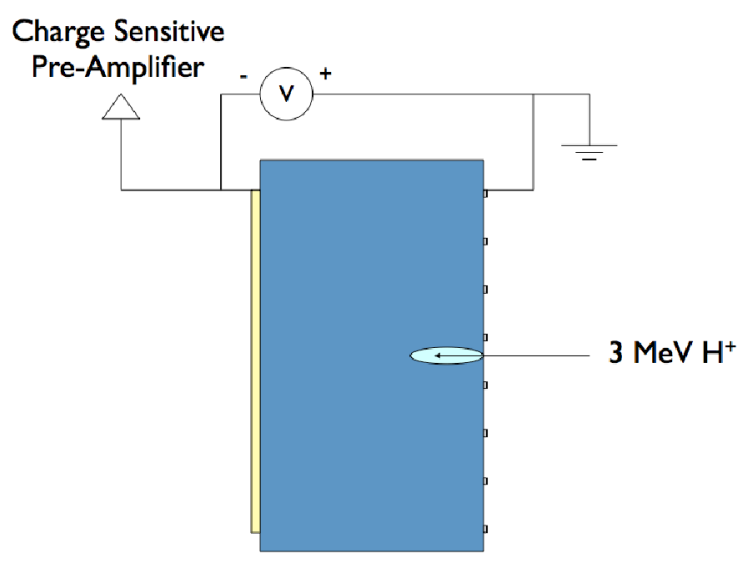

Fig. 3. Measurement of charge collection effeciency.

\section{References}

1. F. Gianotti et al., Eur. Phys. J. C 39, (2005) p. 293.

2. A. Mainwood, Semicond. Sci. Technol. 15, (2000) p. R55.

3. J. Hochedez et al., Phys. Stat. Sol. A 181, 2000 p. 141.

4. C. Manfredotti et al., Nucl. Instrum. Methods A $\mathbf{4 1 0}$, 1998 p. 96.

5. D. Kania et al., Diamond Relat. Matter. 2, 1993 p. 1012.

6. C. Bauer et al., Nuclear. Instrum. Methods A 383, 1996 p. 64.

7. L. Ong et al, IBIC analysis of a single crystal diamond radiation detector, currently in preparation. 